\title{
TWO NICE DETERMINANTAL EXPRESSIONS AND A RECURRENCE RELATION FOR THE APOSTOL-BERNOULLI POLYNOMIALS
}

\author{
FENG QI ${ }^{1,2,3}$ AND BAI-NI GUO ${ }^{4}$ \\ ${ }^{1}$ Institute of Mathematics, Henan Polytechnic University, \\ Jiaozuo City, Henan Province, 454010, China \\ ${ }^{2}$ College of Mathematics, Inner Mongolia University for Nationalities, \\ Tongliao City, Inner Mongolia Autonomous Region, 028043, China \\ ${ }^{3}$ Department of Mathematics, College of Science, Tianjin Polytechnic \\ University, Tianjin City, 300387, China \\ E-mail: qifeng618@gmail.com, qifeng618@hotmail.com, qifeng618@qq.com \\ ${ }^{4}$ School of Mathematics and Informatics, Henan Polytechnic University, \\ Jiaozuo City, Henan Province, 454010, China \\ E-mail: bai.ni.guo@gmail.com, bai.ni.guo@hotmail.com
}

\begin{abstract}
In the paper, the authors establish two nice determinantal expressions and a recurrence relation for the Apostol-Bernoulli polynomials.

Key words and Phrases: Apostol-Bernoulli polynomial; determinantal expression; recurrence relation; determinant; derivative of a ratio between two functions.
\end{abstract}

Abstrak. Pada makalah ini, para penulis menyajikan dua pernyataan berbentuk determinan dan sebuah relasi rekurensi untuk suku banyak Apostol-Bernoulli.

Kata kunci: Suku banyak Apostol-Bernoulli, ekspresi berbentuk determinan, relasi rekurensi, determinan, turunan dari rasio dua fungsi.

\section{INTRODUCTION}

It is well-known that the Bernoulli numbers $B_{k}$, the Bernoulli polynomials $B_{k}(u)$, and the Apostol-Bernoulli polynomials $B_{k}(u, z)$ for $k \geq 0$ can be generated respectively by

$$
\frac{x}{e^{x}-1}=\sum_{k=0}^{\infty} B_{k} \frac{x^{k}}{k !}=1-\frac{x}{2}+\sum_{k=1}^{\infty} B_{2 k} \frac{x^{2 k}}{(2 k) !}, \quad|x|<2 \pi,
$$

2000 Mathematics Subject Classification: Primary 11B68; Secondary 11B83, 11C20, 15A15, 26A06, 26A09, 33B10.

Received: 01-08-2016, revised: 03-03-2017, accepted: 04-03-2017. 


$$
\frac{x e^{u x}}{e^{x}-1}=\sum_{k=0}^{\infty} B_{k}(u) \frac{x^{k}}{k !}, \quad|x|<2 \pi
$$

and

$$
\frac{x e^{u x}}{z e^{x}-1}=\sum_{k=0}^{\infty} B_{k}(u, z) \frac{x^{k}}{k !}, \quad|x|< \begin{cases}2 \pi, & z=1 \\ |\ln z|, & z \neq 1 .\end{cases}
$$

It is clear that these notions have the relations

$$
B_{k}=B_{k}(0) \quad \text { and } \quad B_{k}(u)=B_{k}(u, 1) .
$$

In 1, 2, Apostol connected special values of the Lerch zeta functions with the Apostol-Bernoulli polynomials $B_{k}(u, z)$. In [8, Luo gave a relation between the $\lambda$-power sums and the Apostol-Bernoulli polynomials $B_{k}(u, z)$, which generalize J. Bernoulli's formula on the representation of power sums in terms of the Bernoulli polynomials $B_{k}(u)$. In [7, Kim and $\mathrm{Hu}$ obtained the sums of products identity for the Apostol-Bernoulli numbers $B_{k}(u, z)$, which is an analogue of the classical sums of products identity for the Bernoulli numbers $B_{k}$ dating back to Euler.

Let $p=p(x)$ and $q=q(x) \neq 0$ be two differentiable functions. Then

$$
\frac{\mathrm{d}^{k}}{\mathrm{~d} z^{k}}\left[\frac{p(x)}{q(x)}\right]=\frac{(-1)^{k}}{q^{k+1}}\left|\begin{array}{ccccc}
p & q & 0 & \ldots & 0 \\
p^{\prime} & q^{\prime} & q & \ldots & 0 \\
p^{\prime \prime} & q^{\prime \prime} & 2 q^{\prime} & \ldots & 0 \\
\ldots \ldots \ldots \ldots \ldots & \ldots \ldots \ldots \ldots \ldots & \ldots \ldots \ldots \\
p^{(k-1)} & q^{(k-1)} & \left(\begin{array}{c}
k-1 \\
k
\end{array}\right) q^{(k-2)} & \ldots & q \\
p^{(k)} & q^{(k)} & \left(\begin{array}{c}
k \\
1
\end{array}\right) q^{(k-1)} & \ldots & \left(\begin{array}{c}
k \\
k-1
\end{array}\right) q^{\prime}
\end{array}\right|, \quad k \geq 0
$$

See [3, p. 40]. We can rewrite the formula $(2)$ as

$$
\frac{\mathrm{d}^{k}}{\mathrm{~d} x^{k}}\left[\frac{p(x)}{q(x)}\right]=\frac{(-1)^{k}}{q^{k+1}(x)}\left|W_{(k+1) \times(k+1)}(x)\right|,
$$

where $\left|W_{(k+1) \times(k+1)}(x)\right|$ denotes the determinant of the $(k+1) \times(k+1)$ matrix

$$
W_{(k+1) \times(k+1)}(x)=\left(U_{(k+1) \times 1}(x) \quad V_{(k+1) \times k}(x)\right),
$$

the quantity $U_{(k+1) \times 1}(x)$ is a $(k+1) \times 1$ matrix whose elements $u_{\ell, 1}(x)=p^{(\ell-1)}(x)$ for $1 \leq \ell \leq k+1$, and $V_{(k+1) \times k}(x)$ is a $(k+1) \times k$ matrix whose elements

$$
v_{i, j}(x)= \begin{cases}\left(\begin{array}{l}
i-1 \\
j-1
\end{array}\right) q^{(i-j)}(x), & i-j \geq 0 \\
0, & i-j<0\end{cases}
$$

for $1 \leq i \leq k+1$ and $1 \leq j \leq k$. For more information, please refer to related texts in the recently published papers $[6,9,10,11,12,13,14,15,16,17,19,20,21,22$, [23, 25, 26] and the closely related references therein.

The determinant expressions for the classical Bernoulli polynomials $B_{k}(u)$ have a long history, see [5, p. 53]. 
Applying the formula (3) to $p(x)=1$ and $q(x)=\int_{0}^{1} e^{x(s-u)} \mathrm{d} s$, Qi and Chapman [13, Theorem 1.2] obtained the determinantal expressions

$$
B_{k}(u)=(-1)^{k}\left|\frac{1}{\ell+1}\left(\begin{array}{c}
\ell+1 \\
m
\end{array}\right)\left[(1-u)^{\ell-m+1}-(-u)^{\ell-m+1}\right]\right|_{1 \leq \ell \leq k, 0 \leq m \leq k-1}
$$

and

$$
B_{k}=(-1)^{k}\left|\frac{1}{\ell+1}\left(\begin{array}{c}
\ell+1 \\
m
\end{array}\right)\right|_{1 \leq \ell \leq k, 0 \leq m \leq k-1},
$$

where $|\cdot|_{1 \leq \ell \leq k, 0 \leq m \leq k-1}$ denotes a $k \times k$ determinant.

Influenced by the paper [13], Hu and Kim [6, Theorem 1.10] established

$$
B_{k+1}(u, z)=\frac{(-1)^{k}(k+1)}{(z-1)^{k+1}}\left|\left(\begin{array}{c}
\ell \\
m
\end{array}\right)\left[z(z-u)^{\ell-m}-(-u)^{\ell-m}\right]\right|_{1 \leq \ell \leq k, 0 \leq m \leq k-1}
$$

and

$$
B_{k+1}(z)=\frac{(-1)^{k}(k+1)}{(z-1)^{k+1}}\left|\left(\begin{array}{c}
\ell \\
m
\end{array}\right)\left(z-\delta_{\ell, m}\right)\right|_{1 \leq \ell \leq k, 0 \leq m \leq k-1}
$$

for $k \in \mathbb{N}$ and $z \neq 1$, where

$$
\delta_{\ell, m}= \begin{cases}1, & \ell=m \\ 0, & \ell \neq m\end{cases}
$$

denotes the Kronecker delta. When deriving (4) and (5), $\mathrm{Hu}$ and $\mathrm{Kim}$ used the Leibnitz theorem for differentiation of a product and applied the formula (3) to $p(x)=1$ and $q(x)=z e^{(1-u) x}-e^{-u x}$.

In this paper, we will apply the formula (3) again to establish two nice determinantal expressions and consequently derive a recurrence relation of the ApostolBernoulli polynomials $B_{k}(u, z)$ for $k \in \mathbb{N}$ and $z \neq 1$.

\section{MAIN RESUltS AND THEIR PROOFS}

Our main results, two nice determinantal expressions and a recurrence relation of the Apostol-Bernoulli polynomials $B_{k}(u, z)$ for $k \in \mathbb{N}$ and $z \neq 1$, can be stated as the following theorem.

Theorem 2.1. The Apostol-Bernoulli polynomials $B_{k}(u, z)$ for $k \in \mathbb{N}$ and $z \neq 1$ can be determinantally expressed by

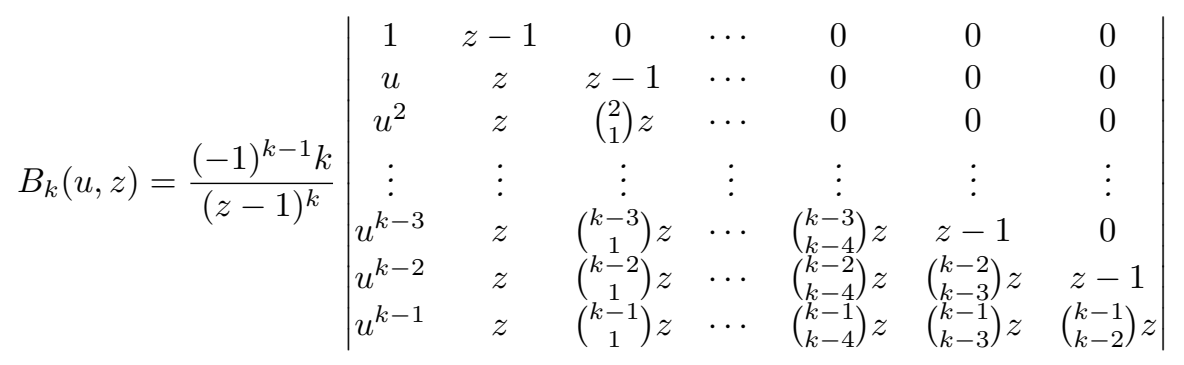


and

$$
B_{k}(u, z)=\frac{(-1)^{k+1}}{(z-1)^{k}}\left|\begin{array}{cccccc}
1 & z-1 & 0 & \cdots & 0 & 0 \\
2 u & \left(\begin{array}{c}
2 \\
1
\end{array}\right) z & z-1 & \cdots & 0 & 0 \\
3 u^{2} & \left(\begin{array}{c}
3 \\
1
\end{array}\right) z & \left(\begin{array}{c}
3 \\
2
\end{array}\right) z & \cdots & 0 & 0 \\
\vdots & \vdots & \vdots & \vdots & \vdots & \vdots \\
(k-2) u^{k-3} & \left(\begin{array}{c}
k-2 \\
k-1
\end{array}\right) z & \left(\begin{array}{c}
k-2 \\
2
\end{array}\right) z & \cdots & z-1 & 0 \\
(k-1) u^{k-2} & \left(\begin{array}{c}
1 \\
1
\end{array}\right) z & \left(\begin{array}{c}
k-1 \\
2
\end{array}\right) z & \cdots & \left(\begin{array}{c}
k-1 \\
k-2
\end{array}\right) z & z-1 \\
k u^{k-1} & \left(\begin{array}{c}
k \\
1
\end{array}\right) z & \left(\begin{array}{c}
k \\
2
\end{array}\right) z & \cdots & \left(\begin{array}{c}
k \\
k-2
\end{array}\right) z & \left(\begin{array}{c}
k \\
k-1
\end{array}\right) z
\end{array}\right|
$$

Consequently, the Apostol-Bernoulli polynomials $B_{k}(u, z)$ for $k \in \mathbb{N}$ and $z \neq 1$ satisfy the recurrence relation

$$
B_{k}(u, z)=\frac{z}{1-z}\left[k B_{k-1}(u, z)+\sum_{r=1}^{k-2}\left(\begin{array}{l}
k \\
r
\end{array}\right) B_{r}(u, z)-\frac{k u^{k-1}}{z}\right], \quad k \geq 2 .
$$

Proof of Theorem 2.1. Applying the formula (3) to $p(x)=x e^{u x}$ and $q(x)=z e^{x}-1$ for $z \neq 1$ gives

$$
u_{\ell, 1}(x)=\left(x e^{u x}\right)^{(\ell-1)} \rightarrow(\ell-1) u^{\ell-2}, \quad x \rightarrow 0
$$

for $\ell \geq 1$ and

$$
v_{i, j}(x)=\left\{\begin{array} { l l } 
{ ( \begin{array} { l } 
{ i - 1 } \\
{ j - 1 }
\end{array} ) ( z e ^ { x } - 1 ) ^ { ( i - j ) } , } & { i - j \geq 0 } \\
{ 0 , } & { i - j < 0 }
\end{array} \rightarrow \left\{\begin{array}{ll}
z-1, & i-j=0 \\
\left(\begin{array}{l}
i-1 \\
j-1
\end{array}\right) z, & i-j>0 \\
0, & i-j<0
\end{array}\right.\right.
$$

as $x \rightarrow 0$ for $i, j \geq 1$. As a result, the Apostol-Bernoulli polynomials $B_{k}(u, z)$ for $k \geq 1$ can be expressed as

$$
=\frac{(-1)^{k}}{(z-1)^{k+1}}\left|\begin{array}{ccccccc}
0 & B_{k}(u, z)=\lim _{x \rightarrow 0} \frac{\mathrm{d}^{k}}{\mathrm{~d} x^{k}}\left(\frac{x e^{u x}}{z e^{x}-1}\right) \\
0 & z-1 & 0 & \cdots & 0 & 0 & 0 \\
2 u & z & z-1 & \cdots & 0 & 0 & 0 \\
\vdots & \vdots & \left(\begin{array}{c}
2 \\
1
\end{array}\right) z & \cdots & 0 & 0 & 0 \\
\vdots & \vdots & \vdots & \vdots & \vdots \\
(k-2) u^{k-3} & z & \left(\begin{array}{c}
k-2 \\
k-2
\end{array}\right) & \cdots & \left(\begin{array}{c}
k-2 \\
k-3
\end{array}\right) z & z-1 & 0 \\
(k-1) u^{k-2} & z & \left(\begin{array}{c}
k-1 \\
1
\end{array}\right) z & \cdots & \left(\begin{array}{c}
k-1 \\
k
\end{array}\right) z & \left(\begin{array}{c}
k-1 \\
k-2
\end{array}\right) z & z-1 \\
k u^{k-1} & z & \left(\begin{array}{c}
k \\
1
\end{array}\right) z & \cdots & \left(\begin{array}{c}
k \\
k-3
\end{array}\right) z & \left(\begin{array}{c}
k \\
k-2
\end{array}\right) z & \left(\begin{array}{c}
k \\
k-1
\end{array}\right) z
\end{array}\right|
$$




$$
=\frac{(-1)^{k+1}}{(z-1)^{k}}\left|\begin{array}{ccccccc}
1 & z-1 & 0 & \cdots & 0 & 0 & 0 \\
2 u & \left(\begin{array}{c}
2 \\
1
\end{array}\right) z & z-1 & \cdots & 0 & 0 & 0 \\
3 u^{2} & \left(\begin{array}{c}
3 \\
1
\end{array}\right) z & \left(\begin{array}{l}
3 \\
2
\end{array}\right) z & \cdots & 0 & 0 & 0 \\
\vdots & \vdots & \vdots & \vdots & \vdots & \vdots & \vdots \\
(k-2) u^{k-3} & \left(\begin{array}{c}
k-2 \\
1
\end{array}\right) z & \left(\begin{array}{c}
k-2 \\
2
\end{array}\right) z & \cdots & \left(\begin{array}{c}
k-2 \\
k-3
\end{array}\right) z & z-1 & 0 \\
(k-1) u^{k-2} & \left(\begin{array}{c}
k-1 \\
1
\end{array}\right) z & \left(\begin{array}{c}
k-1 \\
2
\end{array}\right) z & \cdots & \left(\begin{array}{c}
k-1 \\
k-3
\end{array}\right) z & \left(\begin{array}{c}
k-1 \\
k-2
\end{array}\right) z & z-1 \\
k u^{k-1} & \left(\begin{array}{c}
k \\
1
\end{array}\right) z & \left(\begin{array}{c}
k \\
2
\end{array}\right) z & \cdots & \left(\begin{array}{c}
k \\
k-3
\end{array}\right) z & \left(\begin{array}{c}
k \\
k-2
\end{array}\right) z & \left(\begin{array}{c}
k \\
k-1
\end{array}\right) z
\end{array}\right|
$$

The determinantal expression (7) is thus proved.

Since $B_{0}(u, z)=0$ for $z \neq 1$, the equation (1) can be rewritten as

$$
\frac{e^{u x}}{z e^{x}-1}=\sum_{k=0}^{\infty} B_{k+1}(u, z) \frac{x^{k}}{(k+1) !}, \quad|x|< \begin{cases}2 \pi, & z=1 \\ |\ln z|, & z \neq 1\end{cases}
$$

This implies that

$$
\frac{B_{k+1}(u, z)}{k+1}=\lim _{x \rightarrow 0} \frac{\mathrm{d}^{k}}{\mathrm{~d} x^{k}}\left(\frac{e^{u x}}{z e^{x}-1}\right), \quad k \geq 0 .
$$

Further applying the formula (3) to $p(x)=e^{u x}$ and $q(x)=z e^{x}-1$ for $z \neq 1$ gives

$$
u_{\ell, 1}(x)=\left(e^{u x}\right)^{(\ell-1)} \rightarrow u^{\ell-1}, \quad x \rightarrow 0
$$

for $\ell \geq 1$ and $(9)$. Therefore, we have

$$
\frac{B_{k+1}(u, z)}{k+1}=\frac{(-1)^{k}}{(z-1)^{k+1}}\left(\begin{array}{ccccccc}
1 & z-1 & 0 & \cdots & 0 & 0 & 0 \\
u & z & z-1 & \cdots & 0 & 0 & 0 \\
u^{2} & z & \left(\begin{array}{c}
2 \\
1
\end{array}\right) z & \cdots & 0 & 0 & 0 \\
\vdots & \vdots & \vdots & \vdots & \vdots & \vdots & \vdots \\
u^{k-2} & z & \left(\begin{array}{c}
k-2 \\
1
\end{array}\right) z & \cdots & \left(\begin{array}{c}
k-2 \\
k-3
\end{array}\right) z & z-1 & 0 \\
u^{k-1} & z & \left(\begin{array}{c}
k-1 \\
1
\end{array}\right) z & \cdots & \left(\begin{array}{c}
k-1 \\
k-3
\end{array}\right) z & \left(\begin{array}{c}
k-1 \\
k-2
\end{array}\right) z & z-1 \\
k & z & \left(\begin{array}{c}
k \\
k
\end{array}\right) z & \cdots & \left(\begin{array}{c}
k \\
k-3
\end{array}\right) z & \left(\begin{array}{c}
k \\
k-2
\end{array}\right) z & \left(\begin{array}{c}
k \\
k-1
\end{array}\right) z
\end{array} \mid\right.
$$

for $k \geq 0$. The determinantal expression $(6)$ is thus proved.

Let $M_{0}=1$ and

$$
M_{n}=\left|\begin{array}{cccccc}
m_{1,1} & m_{1,2} & 0 & \ldots & 0 & 0 \\
m_{2,1} & m_{2,2} & m_{2,3} & \ldots & 0 & 0 \\
m_{3,1} & m_{3,2} & m_{3,3} & \ldots & 0 & 0 \\
\vdots & \vdots & \vdots & \vdots & \vdots & \vdots \\
m_{n-2,1} & m_{n-2,2} & m_{n-2,3} & \ldots & m_{n-2, n-1} & 0 \\
m_{n-1,1} & m_{n-1,2} & m_{n-1,3} & \ldots & m_{n-1, n-1} & m_{n-1, n} \\
m_{n, 1} & m_{n, 2} & m_{n, 3} & \ldots & m_{n, n-1} & m_{n, n}
\end{array}\right|
$$


for $n \in \mathbb{N}$. In [4, p. 222, Theorem], it was proved that the sequence $M_{n}$ for $n \geq 0$ satisfies $M_{1}=m_{1,1}$ and

$$
M_{n}=m_{n, n} M_{n-1}+\sum_{r=1}^{n-1}(-1)^{n-r} m_{n, r}\left(\prod_{j=r}^{n-1} m_{j, j+1}\right) M_{r-1}, \quad n \geq 2 .
$$

See also [17, Lemma 2], [18, Lemma 5], 24, Lemma 2], and [25, Remark 3]. Applying this conclusion to determinants in (6) and (7) readily produces the same recurrence relation (8) respectively. The proof of Theorem 2.1 is complete.

Remark 2.1. This paper is a slightly modified version of the preprint [1].

Acknowledgements. The authors appreciate the anonymous referees for their careful corrections to and valuable comments on the original version of this paper.

\section{REFERENCES}

[1] T. M. Apostol, Addendum to 'On the Lerch zeta function', Pacific J. Math. 2 (1952), 10-10; Available online at http://projecteuclid.org/euclid.pjm/1103051938

[2] T. M. Apostol, On the Lerch zeta function Pacific J. Math. 1 (1951), 161-167; Available online at http://projecteuclid.org/euclid.pjm/1103052188

[3] N. Bourbaki, Functions of a Real Variable, Elementary Theory, Translated from the 1976 French original by Philip Spain. Elements of Mathematics (Berlin). Springer-Verlag, Berlin, 2004; Available online at http://dx.doi.org/10.1007/978-3-642-59315-4

[4] N. D. Cahill, J. R. D'Errico, D. A. Narayan, and J. Y. Narayan, Fibonacci determinants, College Math. J. 3 (2002), 221-225; Available online at http://dx.doi.org/10.2307/1559033.

[5] J. W. L. Glaisher, Expressions for Laplace's coeffcients, Bernoullian and Eulerian numbers, etc., as determinants, Messenger Math. (2) 6 (1875), 49-63.

[6] S. Hu and M.-S. Kim, Two closed forms for the Apostol-Bernoulli polynomials, arXiv preprint (2015), available online at http://arxiv.org/abs/1509.04190

[7] M.-S. Kim and S. Hu, Sums of products of Apostol-Bernoulli numbers, Ramanujan J. 28 (2012), no. 1, 113-123; Available online at http://dx.doi.org/10.1007/s11139-011-9340-z

[8] Q.-M. Luo, The multiplication formulas for the Apostol-Bernoulli and Apostol-Euler polynomials of higher order, Integral Transforms Spec. Funct. 20 (2009), no. 5-6, 377-391; Available online at http://dx.doi.org/10.1080/10652460802564324

[9] F. Qi, A determinantal representation for derangement numbers, Glob. J. Math. Anal. 4 (2016), no. 3, 17-17; Available online at http://dx.doi.org/10.14419/gjma.v4i3.6574

[10] F. Qi, Derivatives of tangent function and tangent numbers, Appl. Math. Comput. 268 (2015), 844-858; Available online at http://dx.doi.org/10.1016/j.amc.2015.06.123

[11] F. Qi, Two nice determinantal expressions for the Apostol-Bernoulli polynomials, ResearchGate Working Paper (2016), available online at http://dx.doi.org/10.13140/RG.2. 2.33797.76004

[12] F. Qi, V. Čerňanová, and Y. S. Semenov, On tridiagonal determinants and the Cauchy product of central Delannoy numbers, ResearchGate Working Paper (2016), available online at http://dx.doi.org/10.13140/RG.2.1.3772.6967

[13] F. Qi and R. J. Chapman, Two closed forms for the Bernoulli polynomials, J. Number Theory 159 (2016), 89-100; Available online at http://dx.doi.org/10.1016/j.jnt.2015.07.021

[14] F. Qi and B.-N. Guo, A determinantal expression and a recurrence relation for the Euler polynomials, Preprints 2016, 2016100034, 8 pages; Available online at http://dx.doi.org/ 10.20944/preprints201610.0034.v1

[15] F. Qi and B.-N. Guo, Explicit and recursive formulas, integral representations, and properties of the large Schröder numbers, Kragujevac J. Math. 41 (2017), no. 1, 121-141. 
[16] F. Qi and B.-N. Guo, Expressing the generalized Fibonacci polynomials in terms of a tridiagonal determinant, Matematiche (Catania) $\mathbf{7 2}$ (2017), no. 1, in press.

[17] F. Qi and B.-N. Guo, Some determinantal expressions and recurrence relations of the Bernoulli polynomials, Mathematics 4 (2016), no. 4, Article 65, 11 pages; Available online at http://dx.doi.org/10.3390/math4040065

[18] F. Qi and B.-N. Guo, Some properties of a solution to a family of inhomogeneous linear ordinary differential equations, Preprints 2016, 2016110146, 11 pages; Available online at http://dx.doi.org/10.20944/preprints201611.0146.v1

[19] F. Qi, M. Mahmoud, X.-T. Shi, and F.-F. Liu, Some properties of the Catalan-Qi function related to the Catalan numbers, SpringerPlus (2016), 5:1126, 20 pages; Available online at http://dx.doi.org/10.1186/s40064-016-2793-1.

[20] F. Qi, X.-T. Shi, and B.-N. Guo, Two explicit formulas of the Schröder numbers, Integers 16 (2016), Paper No. A23, 15 pages.

[21] F. Qi, X.-T. Shi, F.-F. Liu, and D. V. Kruchinin, Several formulas for special values of the Bell polynomials of the second kind and applications, J. Appl. Anal. Comput. 7 (2017), no. 3, in press.

[22] F. Qi, J.-L. Wang, and B.-N. Guo, A recovery of two determinantal representations for derangement numbers, Cogent Math. (2016), 3: 1232878, 7 pages; Available online at http: //dx.doi.org/10.1080/23311835.2016.1232878

[23] F. Qi, J.-L. Wang, and B.-N. Guo, A representation for derangement numbers in terms of a tridiagonal determinant, Kragujevac J. Math. 42 (2018), no. 1, 7-14.

[24] F. Qi and J.-L. Zhao, Some properties of the Bernoulli numbers of the second kind and their generating function, J. Differ. Equ. Appl. (2017), in press.

[25] F. Qi, J.-L. Zhao, and B.-N. Guo, Closed forms for derangement numbers in terms of the Hessenberg determinants, Preprints 2016, 2016100035, 11 pages; Available online at http: //dx.doi.org/10.20944/preprints201610.0035.v1

[26] C.-F. Wei and F. Qi, Several closed expressions for the Euler numbers, J. Inequal. Appl. 2015, 2015:219, 8 pages; Available online at http://dx.doi.org/10.1186/s13660-015-0738-9 
